\title{
Molecular signatures of a disturbed nasal barrier function in the primary tissue of Wegener's granulomatosis
}

\author{
M Laudien ${ }^{1,5}$, R Häsler ${ }^{2,5}$, J Wohlers ${ }^{1}$, J Böck ${ }^{2}$, S Lipinski ${ }^{2}$, L Bremer ${ }^{2}$, R Podschun ${ }^{3}$, P Ambrosch ${ }^{1}$, \\ P Lamprecht $^{4}$, P Rosenstiel ${ }^{2}$ and A Till ${ }^{2}$
}

Wegener's granulomatosis (WG) is a complex autoimmune disease of unknown etiology, frequently involving localized inflammation of the nasal mucosa as an early manifestation. The current hypothesis suggests that the disease is triggered by a disturbed interaction between genetic and environmental effects, such as an altered microflora at mucosal layers. In this study, a systematic assessment of 49 transcripts with potential pathophysiological relevance was performed using quantitative real-time PCR in nasal mucosa samples of more than $\mathbf{8 0}$ individuals, including normal control (NC) individuals and disease controls. In addition, colonization with Staphylococcus aureus was quantified in the same individuals to assess its impact on transcriptomic signatures. Transcription profiles show an increased heterogeneity in diseased individuals. In all, 10 transcripts were identified to be differentially expressed $(P \leqslant 0.05$, false discovery rate $\leq 0.05$ ) between patients with $W G$ and $N C$ individuals. These transcripts include antimicrobial peptides (human $\beta$-defensin (DEFB)1: fold-change WG vs. controls: +4.45 , lysozyme: $-3.4, D E F B 4$ and $S 100 A 7$ ( 1100 calciumbinding protein A7): both "switched on" in WG), innate immune receptors (Toll-like receptor 4: -2.1, NOD-like receptor C3: -2.1 , scavenger receptor CD36: +2.9 ), and cytokines (interferon- $\gamma$ : -14 , transforming growth factor- $\beta$ 1: -1.4 , interleukin-17D: -2.7). These transcriptional profiles are independent of $S$. aureus colonization. This study for the first time describes that, on the basis of data obtained from the primary nasal tissue, WG exhibits molecular features that allow its differentiation from other inflammatory disorders with involvement of the nasal mucosa. Further studies based on these findings may enable the identification of subphenotypes, which are currently discussed as an important target for a personalized medicine approach, aiming to reduce side effects and the number of therapy non-responders.

\section{INTRODUCTION}

Wegener's granulomatosis (WG) is a rare, complex antibodyassociated autoimmune small-vessel vasculitis with an incidence of 8 to 12 per $10^{6}$ per year in Northern Europe. ${ }^{1,2}$ In up to $80 \%$ of all patients with WG, the nasal mucosa is involved in the course of the disease, often as the site of primary manifestation ${ }^{3}$ before converting to systemic autoimmune vasculitis associated with highly specific antineutrophil cytoplasmic autoantibodies directed against PR3 (proteinase 3). ${ }^{2}$

Inflammation of the nose and paranasal sinus being a striking feature of WG was already described in 1936 by Wegener. ${ }^{4}$
Epidemiological studies have detected significant higher nasal colonization rates with Staphylococcus aureus in WG patients compared with healthy and disease controls, ${ }^{5}$ and also colonization being a risk factor for relapse of the disease. ${ }^{6-9}$ These data argue for a dysbalanced composition of the nasal microbial flora to be a causative exogenous factor leading to disease manifestation by interacting with a susceptible genetic background, ${ }^{10}$ as described for chronic inflammatory autoimmune processes of the gut and skin (such as Crohn's disease, ulcerative colitis, and atopic eczema ${ }^{11,12}$ ). Another hint for a potential pathophysiological role of $S$. aureus in WG is the

\footnotetext{
${ }^{1}$ Department of Otorhinolaryngology, Head and Neck Surgery, Christian-Albrechts University, Kiel, Germany. ${ }^{2}$ Institute of Clinical Molecular Biology, Christian-Albrechts University, Kiel, Germany. ${ }^{3}$ Institute of Infection Medicine, University Hospital SH, Campus Kiel, Kiel, Germany. ${ }^{4}$ Department of Rheumatology, Vasculitis Center UKSH and Rheumaklinik Bad Bramstedt, University of Lübeck, Lübeck, Germany. ${ }^{5}$ These authors contributed equally to this work. Correspondence: M Laudien (laudien@hno.uni-kiel.de) 
finding of specific adaptive immune responses to $S$. aureus in WG patients. ${ }^{9,13}$

Administration of trimethoprim/sulfamethoxazole (T/S) is successfully used in WG therapy, ${ }^{14-16}$ especially to prophylactically reduce relapse rates in WG in remission. ${ }^{17}$ However, the therapeutic effect is dependent on disease stage as patients with localized WG benefit, whereas patients with generalized WG do not. ${ }^{18,19}$ Owing to high intrinsic resistance rates, antibiotic treatment with $\mathrm{T} / \mathrm{S}$ is usually not the first choice for treating S. aureus infections, ${ }^{20}$ and the positive therapeutic effect does not seem to depend on the influence on nasal S. aureus colonization rates in WG as demonstrated recently. ${ }^{5}$ Mechanisms of the observed beneficial effect of T/S treatment in WG subgroups still remain unclear, particularly as a general immunomodulatory effect of folic acid derivatives is unlikely within the range of used dosages. ${ }^{17,21}$ However, although T/S has a broad activity against both Gram-positive and Gram-negative bacteria, a number of particular taxa such as clostridia and varying proportions of strains of intrinsically sensitive taxa are resistant. Thus, it might be hypothesized that treatment with $\mathrm{T} / \mathrm{S}$ provokes an alteration in the composition of the nasal microflora resulting in positive effects on the disease course independent of $S$. aureus carriage, which might be responsible for successful treatment. This is especially emphasized by the prominent role of mucosal colonization with commensal bacteria, which essentially contribute to the efficacy of the mechanistic and immunological barrier function conveyed by the nasal mucosa. ${ }^{22}$

Higher frequencies of nasal S. aureus colonization rates might also be facilitated, at least partly, by the severely impaired ciliary beat frequency of the airway epithelia in WG, thus leading to disturbed mechanical barrier function as has been recently demonstrated by our group. ${ }^{23}$

Taken together, microbial challenge, particularly S. aureus colonization of the nasal mucosa, is widely discussed to represent the exogenous trigger for WG or at least a major component in disease etiology. Given the complex interplay and mutual impact of mucosal microflora and gene expression by mucosal epithelial cells, as documented by a recent study describing peroxisome proliferator-activated receptor- $\gamma$ dependent gene expression in the colon of patients with Crohn's disease, ${ }^{24}$ we hypothesized that gene expression of the nasal mucosa of WG patients reflects disease pathophysiology when compared with controls. The main objective of this study was to verify this hypothesis by systematically monitoring mRNA expression patterns of genes, which are associated with antimicrobial defense mechanisms and epithelial barrier function. In particular, we focused on genes encoding for receptors and adaptors of innate immune signaling cascades, antimicrobial peptides (AMPs), and cytokines. In addition, genes with proven or assumed relevance to WG etiopathogenesis (such as PAR-2 [proteinase-activated receptor 2], PR3, FN) were included to identify potentially disease-relevant processes.

\section{RESULTS}

\section{Patient characteristics}

WG patients $(n=29)$ were compared with normal control (NC) $(n=27)$ patients and patients with chronic rhinosinusitis with nasal polyps (CRS) $(n=31$, Table 1$)$.

The mean time from first manifestation of WG until study entry was 8 years (range $51-0$ years), and the mean time from the first diagnosis until study entry was 4 years (range 18-0 years).

Signs for systemic inflammation (C-reactive protein, erythrocyte sedimentation rate, white blood cell) were heterogeneous (Table 1).

Nine WG patients and one CRS patient received systemic antibiotics. One WG and one CRS patient received local antibiotic ointment. Further systemic medication was heterogeneous with

Table 1 Patient characteristics

\begin{tabular}{|c|c|c|c|}
\hline & WG $(n=29)$ & CRS (n=27) & $N C(n=31)$ \\
\hline Sex & 15 males, 14 females & 19 males, 8 females & 21 males, 10 females \\
\hline Mean ESR (mm after the first hour) & 36.2 (s.d. 28.5, range 2-90) & 7.7 (s.d. 8.2, range $1-40)$ & 7.1 (s.d. 4 , range $2-15$ ) \\
\hline Mean WBC $\left(\mathrm{nl}^{-1}\right)$ & 8.1 (s.d. 2.9, range 4.1-18.6) & 6.5 (s.d. 1.4, range 3.9-9.3) & 6.8 (s.d. 1.5 , range $4.6-11.2$ ) \\
\hline Median BVAS-1 & 4 & & \\
\hline Median BVAS-2 & 0 & & \\
\hline Median VDI & 1 & & \\
\hline
\end{tabular}

Abbreviations: BVAS, Birmingham Vasculitis Activity Score; CRP, C-reactive protein; CRS, chronic rhinosinusitis with polyps; ESR, erythrocyte sedimentation rate; EULAR, European League against Rheumatism; NC, normal control individuals; VDI, Vasculitis Damage Index; WBC, white blood cell; WG, Wegener's granulomatosis. 
$1 \mathrm{NC}$ individual and 1 patient with CRS receiving steroids for treating asthma, $24 \mathrm{WG}$ patients receiving steroids, 3 receiving cyclophosphamide bolus therapy, 5 oral cyclophosphamide, 9 methotrexate, 4 azathioprine or leflunomide, respectively, and 1 mycophenolate mofetil or rituximab, respectively, in different combinations and dosages. The cumulative cyclophosphamide dosage of 15 patients who received this treatment was $25 \mathrm{~g}$ (s.d. 49.3). The median cytoplasmic antineutrophil cytoplasmic autoantibody level for WG patients was 1:80. In 16 of 29 WG patients (55\%), 12 of $32 \mathrm{NC}$ (37\%), and 12 of 27 CRS patients (44\%), endonasal S. aureus colonization was detected.

In all, 3 WG patients were classified as localized, 2 as early systemic, 22 as generalized, and 2 as refractory according to the subgroup classification by EULAR (The European League against Rheumatism, Table 1).

Referring to the EULAR guidelines, activity was defined as 8 patients in remission, 7 minor, 4 major, 2 refractory, 5 low activity, and 3 responses.

The median BVAS-1 (Birmingham Vasculitis Activity Score, new or worsened activity) was 4 (maximum 31 , minimum 0 ), and the median BVAS-2 (persistent activity) was 0 (maximum 5, minimum 0). The median VDI (Vasculitis Damage Index) was 1 (maximum 4, minimum 0 ). At study entry, $16 \mathrm{WG}$ patients showed involvement of the upper respiratory tract by vasculitis according to the ear, nose, throat, lung, kidney classification (Table 1).

No endonasal activity was endoscopically detected in 16 patients, mild activity was observed in 10 patients, and 2 WG patients showed moderate activity.

\section{Candidate gene selection}

In total, 43 of 49 transcripts were detected in at least 10 samples of NC, WG, or CRS and therefore subjected to subsequent analysis (A complete list of all genes with corresponding fold changes and $P$-values is presented in Supplementary Table S1 online).

\section{Expression analysis}

Applying stringent cutoff criteria (multiple testing correction, $P \leqslant 0.05$ and false discovery rate (FDR) $\leqslant 5 \%$ ), eight transcripts showed significantly differential mRNA expression between WG patients and NCs: CD36 (thrombospondin receptor, leukocyte differentiation antigen CD36), human $\beta$-defensin 1 (DEFB1), interferon- $\gamma$, interleukin-17D, lysozyme (renal amyloidosis, $L Y Z$ ), NOD-like receptor (NLR) family, CARD domain containing 3 (NLRC3), transforming growth factor- $\beta 1$, and Tolllike receptor 4 (TLR4). The detailed nature of the regulation is displayed in Figure 1. In addition, human $\beta$-defensin 2 (DEFB4, formerly referred to as HBD2) and $\$ 100$ calciumbinding protein A7 (S100A7), which were only detected in WG samples, were categorized as significantly "switched on" $\left(P \leqslant 1 \times 10^{-6}\right)$, but the FDR criteria could not be applied because of the lack of signals in control groups. Individual patient traits were excluded as confounding factors on the basis of results of a substratification analysis: All trends and significances observed in the non-stratified cohort remained similar in the substratified cohort (trends: Supplementary Figure S2 online, significance of
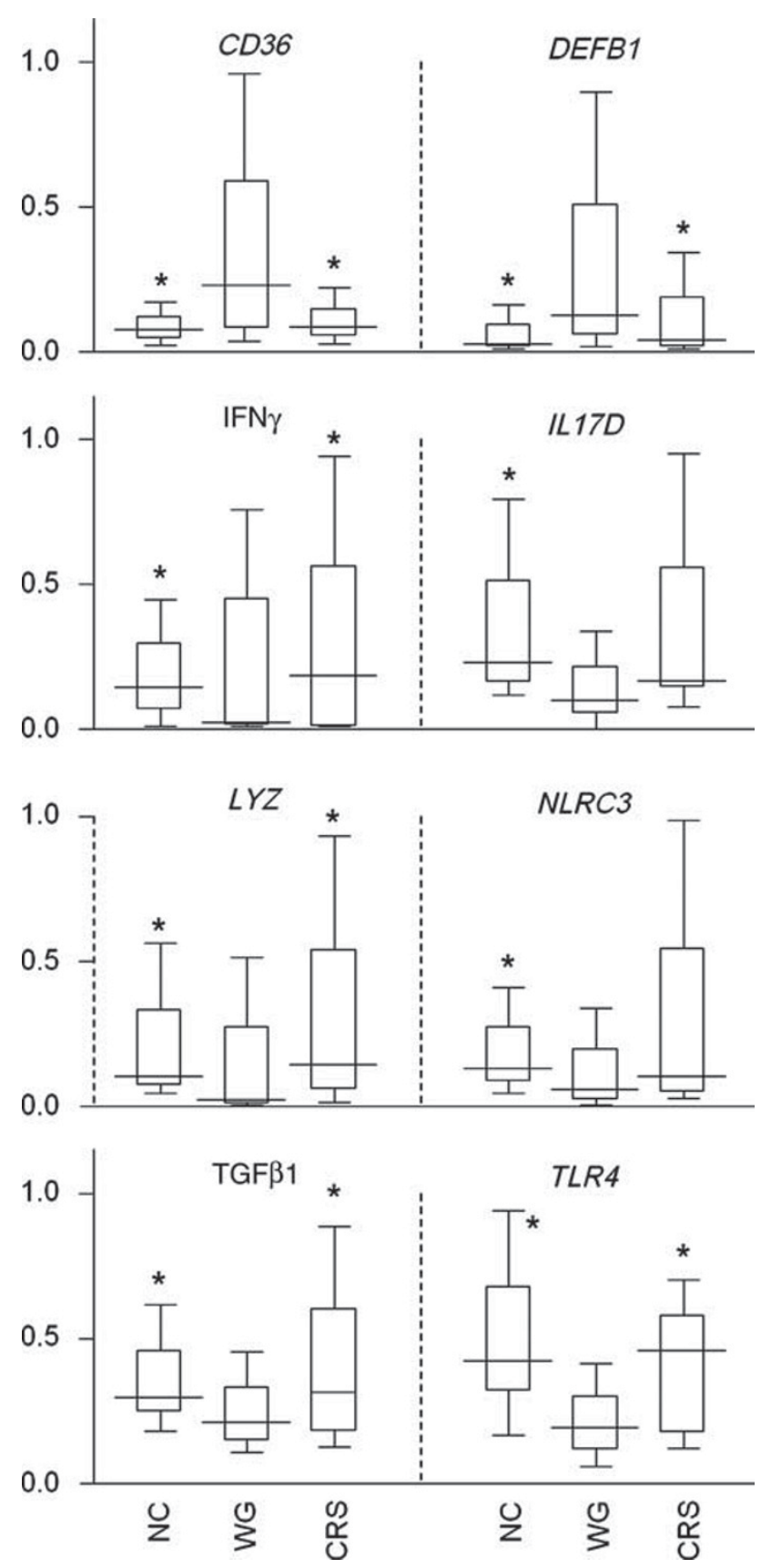

Figure 1 Identified Wegener's granulomatosis (WG) candidate transcripts. Relative mRNA expression of eight significantly regulated transcripts in NC (normal control) individuals, WG patients, and CRS (chronic rhinosinusitis with nasal polyps) patients. Box plots represent the 25th, 50th, and 75th percentile, whereas whisker plots display the 5th and 95th percentile. Asterisks $\left(^{*}\right)$ highlight transcripts which resulted in a $P$-value $\leqslant 0.05$ and false discovery rate of the induction of $\leqslant 5 \%$ when compared with WG. DEFB1, human $\beta$-defensin 1 ; IFN $\gamma$, interferon- $\gamma$; IL17D, interleukin-17D; $L Y Z$, lysozyme; NLRC3, NOD-like receptor C3; TGF $\beta$, transforming growth factor- $\beta$; TLR4, Toll-like receptor-4.

differences: Supplementary Figure S3 online). Substratification resulting in groups containing $\leqslant 11$ individuals showing changes in trends and significances was considered non-relevant because of reduced statistical power. 
$\mathrm{NC}$

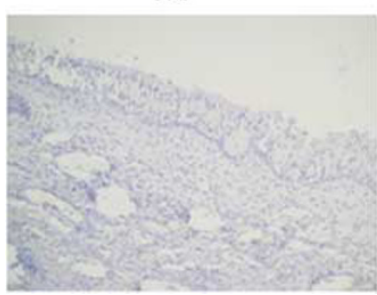

wc

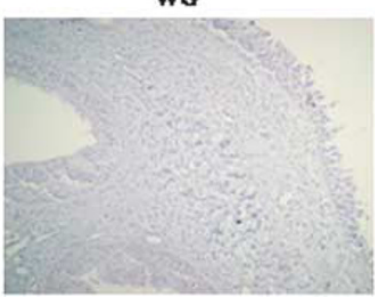

CRS

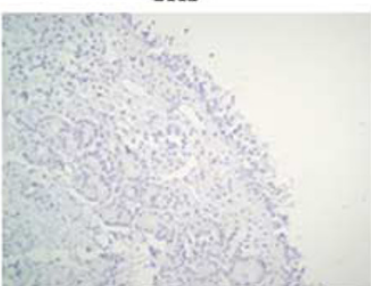

Figure 2 Tissue architecture and cell composition of biopsy specimens. Comparable tissue composition was detected in biopsy specimens of the investigated groups by hematoxylin staining. CRS, chronic rhinosinusitis with nasal polyps; NC, normal control; WG, Wegener's granulomatosis. Magnification: $\times 200$.

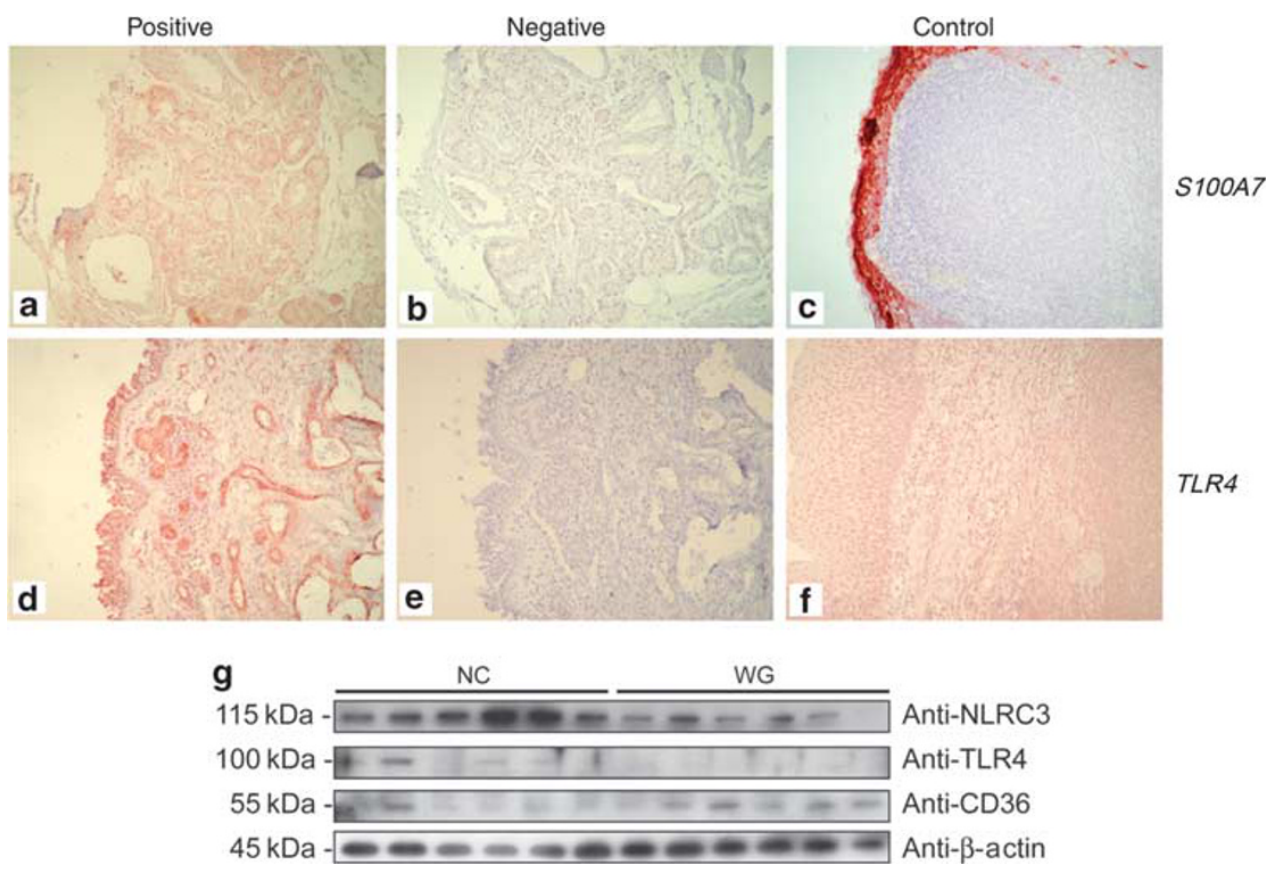

Figure 3 Detection of candidate genes at the protein level. Verification of differential gene expression using immunohistochemical staining: (a) S100A7, (b) S100A7-negative control, (c) S100A7 tonsil, (d) TLR4, (e) TLR4 negative control, and (f) TLR4 tonsil . Magnification: $\times 200$. Verification of differential gene expression using immunoblotting. Protein lysates from patient biopsies ( $n=6$ for NC, $n=6$ for WG) were subjected to SDS-PAGE and probed for protein levels of selected hit genes using specific antibodies. Loading levels and transfer efficiency were demonstrated by detection of $\beta$-actin. NC, normal control; NLRC3, NOD-like receptor C3; S100A7, S100 calcium-binding protein A7; TLR4, Toll-like receptor-4; WG, Wegener's granulomatosis.

\section{Histological analysis of nasal biopsies}

As shown in Figure 2, no significant differences in tissue architecture and cellular composition in WG, NC, and CRS biopsies were observed. In particular, no macroscopic signs of enhanced infiltration of inflammatory cells were detected, which ensured sample homogeneity. Exemplarily, expression patterns of S100A7 and TLR4 could be demonstrated at the protein level by means of immunohistochemistry (12 WG, 6 CRS, and $11 \mathrm{NC}$, Figure 3a). Furthermore, differential expression of cytosolic- or membrane-bound hit genes (such as NLRC3, TLR4, and CD36) could be confirmed at the protein level using immunoblotting on a patient subset ( $n=6$, NC and WG, Figure $3 \mathbf{b}$ ), thus further supporting the validity of our findings.

\section{Principal component analysis}

Using the selected eight candidate transcripts, the principal component analysis resulted in a separation of $\mathrm{NC}$ from WG patients (see also Supplementary Figure S4). In Figure 4, the first two components are plotted. The first component displays a larger variation in WG patients when compared with NCs, whereas the second component displays similar variation for both groups. Importantly, other characteristics of patients and NC individuals did not represent a confounding factor, which was tested in a substratification analysis (substratification based on treated/untreated, age, gender, presence of cytoplasmic antineutrophil cytoplasmic autoantibody/BVAS-1/BVAS-2/VDI/disease extent index, and activity score according to EULAR; data not shown). 


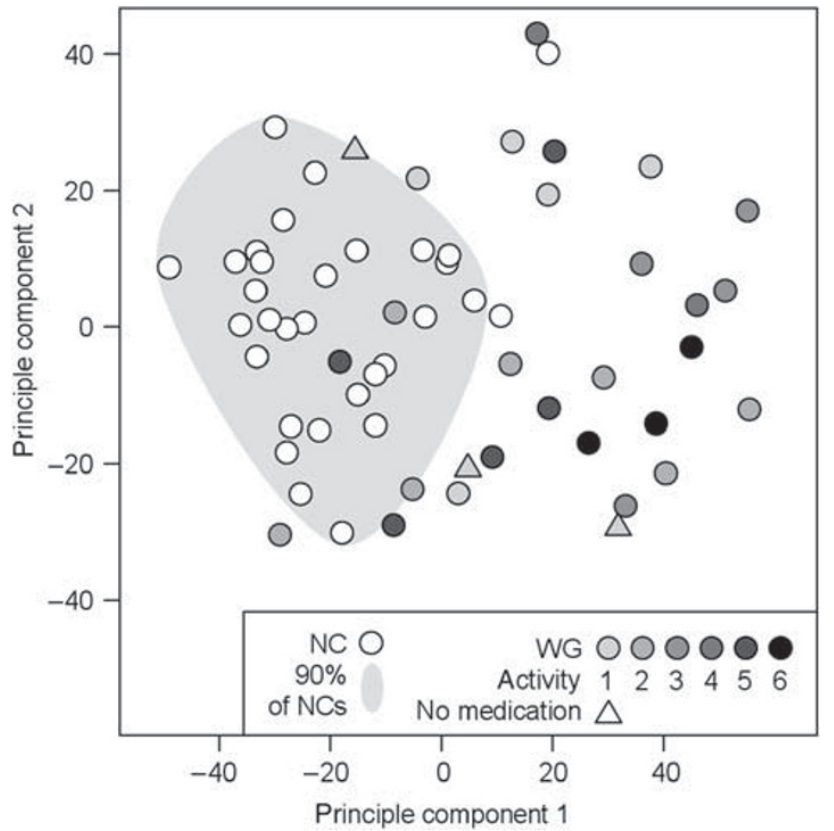

Figure 4 Principal component analysis. The two strongest principal components are plotted ( $x / y$ axis). The principal component analysis is based on eight differentially expressed transcripts as shown in Figure 1. Each circle represents an individual WG patient (gray circles, shaded by disease activity according to EULAR) or a normal control individual (white circles). Untreated WG patients are displayed as triangles. The shaded area encircles $>90 \%$ of all normal control individuals. EULAR, European League against Rheumatism; $\mathrm{NC}$, normal control; WG, Wegener's granulomatosis.

\section{Cluster analysis}

A clustering based on the 8 candidate genes and 61 individuals resulted in distinct grouping of NC and WG patients, while each group contained outliers (Figure 5; see also Supplementary Figure S5).

\section{Correlation analysis}

A gene-to-gene correlation analysis could identify further disease features: A group of closely positively co-regulated genes was found in WG (Supplementary Figure S1A online), NC (Supplementary Figure S1B online), and in CRS patients (Supplementary Figure S1C online). Two transcripts, which were part of the co-regulated gene group in NCs, were not coregulated in WG and CRS (DEFB4 and PGLYRP1).

\section{Substratification by S. aureus colonization}

Substratification of expression data by nasal S. aureus colonization did not lead to additional significances, which were not present before substratification (Supplementary Figures S2 and S3 online).

\section{DISCUSSION}

WG is an antibody-associated vasculitis affecting up to 12 per $10^{6}$ patients per year in Northern Europe. ${ }^{1,2}$ The molecular mechanisms of its pathogenesis, as well as the relevance of pathogens to disease manifestation and progression, are still unclear. Transcriptional profiles together with bacterial profiling provide

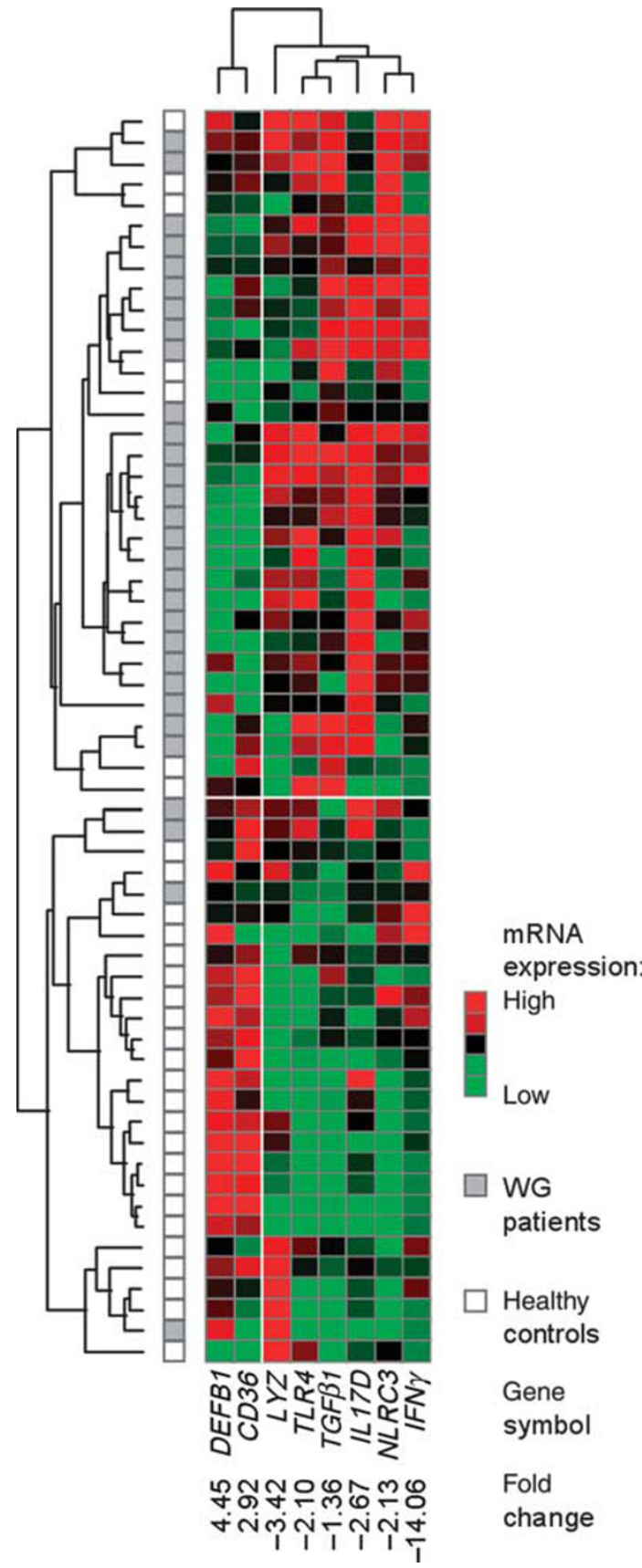

Figure 5 Cluster analysis of candidate transcripts. The cluster is based on the expression values for 8 transcripts in 29 WG patients and in 31 normal control individuals. Each column represents a transcript, whereas each row represents a patient. The row dendrogram illustrates the similarities between patients, and the column dendrogram illustrates the similarities between transcripts. The heatmap is colored according to the relative mRNA expression (green for low mRNA expression, black for middle mRNA expression, and red for high mRNA expression). Fold changes listed are based on the ratios of the medians, $P$-values were calculated using the Mann-Whitney U-test and subsequently corrected for multiple testing using the Benjamini-Hochberg correction. DEFB1, human $\beta$-defensin 1 ; IFN $\gamma$, interferon- $\gamma$; IL17D, interleukin-17D; $L Y Z$, lysozyme; NC, normal control; NLRC3, NOD-like receptor C3; TGF $\beta$, transforming growth factor- $\beta$; TLR4, Toll-like receptor-4; WG, Wegener's granulomatosis. 
a powerful tool for assessing disease-relevant molecular events in vivo. In this study, we aimed to identify gene expression patterns associated with WG by comparing transcriptional profiles of the nasal mucosa of patients suffering from WG with NCs. To this aim, we applied a candidate gene approach analyzing 49 selected target genes involved in epithelial barrier function or with potential or proven role in WG disease pathogenesis. As a disease specificity control, CRS patients were included. Results were confirmed at the protein level using immunohistochemistry and immunoblotting.

\section{Disease-relevant transcriptional profiles}

We identified a number of transcripts that were significantly dysregulated between WG and NCs, as well as between WG and CRS, thus indicating WG-specific transcript signatures and not just a general inflammatory response. Moreover, the significance of the results indicates that these signatures are not the consequence of increased heterogeneity in cell composition, which would have been reflected by stronger, but non-significant, modulations of mRNA levels. The lack of upregulation of typical proinflammatory marker genes (such as IL8, TNF-alpha, and IFN-gamma), as well as the similarity in tissue architecture and cellular composition of biopsy specimens comparing the investigated groups, further emphasize the validity of these signatures as WG-associated molecular profiles in the absence of local inflammatory conditions. The presented study setup does not allow to draw conclusions about individual cell types or infiltrates; however, the complex interplay between cells under inflammatory conditions as reflected by the primary tissue represents a key element of disease pathogenesis as demonstrated earlier. ${ }^{25}$ Signatures display a remarkable overrepresentation of specific AMPs in WG as DEFB1 is significantly upregulated in WG than in controls and, even more striking, both S100A7 and $D E F B 4$ are exclusively detectable in WG but not in controls. In contrast to these AMPs, we found lysozyme to be significantly downregulated in WG. Interestingly, the upregulation of $\beta$-defensins and 100A7 (Psoriasin) is not accompanied by a similar upregulation of genes representing the host cell's sensory machinery, as neither NLRs nor TLRs or their respective adaptors show increased expression levels. Moreover, the lipopolysaccharide-specific receptor, TLR4, and a member of the NLR family, NLRC3, even show highly significant downregulation in WG both at the mRNA and the protein levels. In consequence, the observed increase in AMP expression levels may result either from higher local concentrations of pathogen-associated molecular structures that initiate AMP induction or from dysbalanced gene regulation by still unknown mechanisms. Intriguingly, primary nasal epithelial cell cultures of WG patients respond to challenges with the $S$. aureus supernatant with less secretion of hBD-3, but not of LL-37 compared with controls (unpublished data). Thus, the antimicrobial response of the nasal barrier does not seem to be generally diminished or enhanced with respect to AMP, but substantially dysregulated.

Our data show a significant downregulation of mRNA levels of the cytokines transforming growth factor- $\beta 1$ and interleukin-17D in WG compared with controls. Interestingly, the gene products of these two transcripts show higher protein expression levels in WG and are currently being discussed to be crucially involved in the pathogenesis of WG by mediating immune cell activation, antibacterial responses, and PR3 processing. ${ }^{26-28}$ This discrepancy between cytokine mRNA levels and protein abundance, which has been found in many other complex tissues ${ }^{29}$ may be based on hitherto undefined regulatory processes contributing to cytokine release and thus warrants further investigations.

\section{Increased heterogeneity in diseased transcription patterns}

Several approaches to use personalized medicine have demonstrated that similar macroscopic disease phenotypes often cover a diverse group of underlying molecular phenotypes. ${ }^{30,31}$ The presented results document that transcriptional diversity is increased in diseased individuals, shown by (i) outliers in clustering results and (ii) increased heterogeneity in the WG group in the principal component analysis. Interestingly, neither disease activity nor treatment with immunosuppressants contribute to this heterogeneity as demonstrated by the principal component analysis. These differences may also indicate variations in the complex cell composition of a biopsy; however, microscopical inspection of the biopsies and the non-parametric statistical methods allow the conclusion that WG introduces an increased variation in transcript levels: This observation indicates that a study setup with a larger WG cohort may be able to identify subphenotypes. In the same context, other molecular parameters, such as high-resolution bacterial profiles, could also lead to the identification of subphenotypes. Nevertheless, it is important to note that the clinical and molecular heterogeneity, as well as interindividual differences between cases may conceal molecular signatures characteristic of disease subphenotypes. The current study setup does not allow identification of such subphenotypes. In contrast, the presented results represent molecular patterns that are common to the entire group of diseased individuals, regardless of potential subphenotypes.

Interestingly, the largest part of heterogeneity between WG individuals is contributed by one single transcript ( $>99 \%$ by $L Y Z$, as quantified by its variation coefficient), whereas all other seven identified candidate transcripts display a much lower variation. This observation may indicate that the transcripts are controlled by different mechanisms, such as genetic control and environmental control. ${ }^{32}$

\section{Impact of S. aureus on transcriptional profiles}

As nasal colonization with $S$. aureus has repeatedly been linked to WG susceptibility and relapse rate, we analyzed whether the observed transcriptional alterations were correlated with S. aureus carriage. To our surprise, substratification for S. aureus carriage did not result in improvement in significance levels but reduced the number of significantly dysregulated transcripts. This finding seems to be contrary to the general assumption that $S$. aureus colonization might represent a pathological trigger that a priori initiates general immune responses, which ultimately manifest in WG pathologies. An alternative explanation would argue for a secondary role of S. aureus colonization, 
i.e., a pathophysiological situation in which nasal colonization represents a consequence of initially disturbed nasal barrier function rather than a causative event that initiates the pathological cascade. Moreover, it is tempting to speculate that the lack of differences between $S$. aureus carriers and non-carriers in steroid-treated WG patients indicates that other elements of the microbiota have more impact on disease pathophysiology. In this context, it is noteworthy that lysozyme, which displays strong downregulation in WG in our data set, has been demonstrated to exert effective antibacterial activity against a specific subset of $S$. aureus strains. ${ }^{33}$ Thus, it is tempting to speculate that impaired lysozyme expression in WG might favor colonization with these $S$. aureus strains compared with healthy individuals. In consequence, the significant upregulation of $\beta$-defensin family members, which have also been shown to be active against S. aureus, ${ }^{34}$ might represent a direct antibacterial response and an attempt to restore immunological barrier function. However, as our current experimental setup does not allow to distinguish between causality and consequence of $S$. aureus in the described pathological setting, future studies are justified, which will focus on this intriguing aspect in detail. However, the possibility that other microorganisms may be responsible for the assumed early triggering event is also not excluded. Consequently, a higherresolution picture of the microbial community residing in the nasal cavity of healthy and diseased individuals suffering from chronic inflammatory or autoimmune-mediated disorders is required. Interestingly, molecular profiling of the microbial communities residing in the gastrointestinal tract has revealed that reduced diversity of the bacterial microflora is indicative of chronic inflammatory bowel diseases, which has often served as a model disease for other inflammatory barrier disorders. ${ }^{35-37}$ According to these findings, it is hypothesized that a significant shift in the delicately balanced composition of intestinal communities might activate host responses that result in the initiation of inflammatory conditions and/or establishment of chronicity. Similar to inflammatory bowel disease, WG is considered to belong to the group of multifactorial or complex diseases, which are generally believed to originate from a complex interplay between environmental trigger factors and genetic background. ${ }^{11}$ As WG also shares some clinical features with inflammatory bowel disease entities such as Crohn's disease (e.g., chronic inflammation, granuloma formation, involvement of epithelial layers, potential bacterial trigger) and given the data represented here, further studies are warranted to characterize the involvement of bacterial colonization in the pathogenesis of WG.

\section{Complexity of genome-transcriptome interactions}

Despite the obvious difficulties, regarding statistical power caused by the low incidence of WG, a number of recent studies has led to the identification of genetic variants representing risk factors for WG. ${ }^{38}$ With respect to these findings, our data presented here warrant the hypothesis that a specific genetic or epigenetic background contributes to alterations of mucosal transcriptional profiles, which enhance the individual's susceptibility to WG after encountering a triggering event. Recently, genetic variations of interferon-regulatory factor 5 have been demonstrated to be linked to WG susceptibility. ${ }^{39}$ As interferonregulatory factor 5 represents a major downstream regulator of the TLR-MyD88 signaling pathway mediating release of proinflammatory cytokines, ${ }^{40}$ it would be interesting to characterize the role of this variation (or that of other newly identified target genes) in the transcriptional regulation of the WG-specific genes displayed here. A combined analysis of genetic risk profile and transcriptional signatures might thus ultimately lead to establishment of a diagnostic procedure based on molecular signatures that could be beneficial for efficient diagnosis. The ultimate goal would be to establish a molecular diagnosis procedure supporting classical clinical diagnosis aiming to identify subphenotypes and to differentiate responders from non-responders in a given therapy context. ${ }^{41-45}$

It is important to note that the presented results do not document WG-specific effects exclusively. In contrast to the eight selected candidate transcripts which indicate WG specificity, a co-regulation analysis revealed high similarities between WG and CRS transcript patterns differentiating both from NC individuals. This suggests the presence of specific and shared mechanisms when comparing two distinct pathophysiological processes affecting the same tissue.

\section{Conclusions}

Altered responses to pathophysiological events can lead to an imbalanced immune response. This study for the first time shows potentially disease-relevant mRNA expression patterns that were generated by analyzing the primary WG tissue. Our main findings demonstrate that the molecular profiles in WG differ from those observed in other inflammatory defects in the same tissue. Future studies targeting subphenotypes based on transcriptional and bacterial profiling may help to provide a directed personalized therapeutic approach, thus reducing side effects and the number of non-responders.

\section{METHODS}

Patients and biopsies. From February till November 2008, 29 WG patients, $31 \mathrm{NC}$ individuals, and 27 disease controls (patients suffering from CRS) were included in this study (Table 1). Biopsies were obtained from the mucosa of turbinates. For RNA isolation, biopsies were immediately frozen in liquid nitrogen and stored at $-80^{\circ} \mathrm{C}$ until use. For immunohistochemistry, 29 biopsies ( $12 \mathrm{WG}, 6 \mathrm{CRS}$, and $11 \mathrm{NC}$ ) were fixed in $4 \%$ formalin and embedded in paraffin.

The study was approved by the ethics committee of the University of Kiel, Germany, and participants gave written informed consent (AZ A101/07). Exclusion criteria included pregnancy, anticoagulant treatment, and under-age.

The presence of systemic inflammation was assessed by analysis of the following parameters: serum levels of C-reactive protein (measured using a conventional C-reactive protein assay (Roche Diagnostics, Mannheim, Germany)), erythrocyte sedimentation rate, and white blood cell count.

WG was diagnosed in accordance with the Chapel Hill definitions and the ACR (American College of Rheumatology) classification criteria for WG as recommended by EULAR. ${ }^{46}$ In $59 \%$ of cases (17 of 29 patients), WG was biopsy proven (nasal mucosa, lung, kidney). The vast majority of these WG-positive biopsies were taken endonasally (65\%, 11 of 17). Disease activity was measured using BVAS, organ damage as 
a consequence of granulomatous inflammation and vasculitis using the $\mathrm{VDI},{ }^{47}$ and organ involvement was assessed by ELK classification. ${ }^{48} \mathrm{WG}$ subgroups (localized, early systemic, and generalized WG), relapse and remission, as well as activity was diagnosed and defined according to the EUVAS (European Vasculitis Study Group) definitions and the recent EULAR recommendations. ${ }^{46}$ All patients were subjected to a standardized interdisciplinary evaluation as described earlier and examined endoscopically as proposed by Paulsen and Rudert ${ }^{49}$ by an ENT surgeon to assess WG activity. ${ }^{50}$ Biopsies of the inferior turbinates were taken from places without any macroscopic disease activity (e.g., granuloma, edema, bloody patches, purulent secretion, crusts) to ensure the lack of immune cell infiltrates.

NCs had no anamnestic pathology of the nasal or paranasal mucosa and were examined endoscopically and microscopically both preoperatively and intraoperatively. Biopsies were taken while performing airway passage improving surgery. NCs were included or excluded after the operational procedure.

CRS patients were diagnosed according to the criteria of the American Academy of Otolaryngology-Head and Neck Surgery, the American Academy of Otolaryngic Allergy, the American Rhinologic Society, and the Sinus and Allergy Health Partnership, ${ }^{51}$ and biopsies were obtained by performing functional endoscopic sinus surgery.

Analysis of nasal colonization with $\boldsymbol{S}$. aureus. Both anterior nostrils were swabbed after a standard operational procedure. The cotton swab was transferred into a sterile tube and incubated for $45 \mathrm{~min}$ at room temperature under rotation in $10 \mathrm{~mm}$ sodium phosphate buffer with $0.1 \%$ bovine serum albumin. In all, $100 \mu$ of the swab supernatant was plated onto Chapman Agar for quantification of $S$. aureus colonization. Furthermore, enrichment cultures were performed by incubating the swab tip in tryptic soy broth overnight at $37^{\circ} \mathrm{C}$. Isolates obtained were identified as $S$. aureus by typical appearance of the colonies, hemolysis, expression of clumping factor and protein A, the ability to coagulate citrate plasma, and by acid production from mannitol fermentation.

RNA isolation and cDNA synthesis. Total RNA was isolated from snapfrozen nasal biopsies using a commercial kit (RNeasy, Qiagen, Hilden, Germany). For quantification of mRNA levels of 49 selected target genes and 3 housekeeping genes, cDNA was synthesized from $800 \mathrm{ng}$ of total RNA using the Advantage RT-for-PCR kit (Clontech Laboratories, Palo Alto, CA) with oligo-dT-primers according to the manufacturer's protocol. RNA was checked for genomic contamination using standard PCR protocols (AmpliTaq DNA Polymerase, Applied Biosystems, Foster City, CA), with primers specific for the housekeeping gene GAPDH (GAPDH-forward CCAGCCGAGCCACATCGC, GAPDH-reverse ATGAGCCCCAGCCTTCTCCAT) and the following program: 1 hold of $94^{\circ} \mathrm{C}$ for $5 \mathrm{~min}, 40 \mathrm{cycles}$ of $94^{\circ} \mathrm{C}$ for $30 \mathrm{~s}, 54^{\circ} \mathrm{C}$ for $30 \mathrm{~s}, 72^{\circ} \mathrm{C}$ for $60 \mathrm{~s}$, and 1 hold of $72^{\circ} \mathrm{C}$ for $7 \mathrm{~min}$. The resulting cDNA samples were checked for successful cDNA synthesis using the same primers and slightly modified PCR procedures (30-35 cycles). Resulting amplicons were resolved on $2 \%$ agarose gels stained with ethidium bromide and visualized through an ultraviolet light digital imaging system. Only samples passing the quality criteria (absence of genomic DNA contamination, intact cDNA) were used for further analyses.

Selection of target genes. Given the low incidence rate of WG and the weak statistical power of a large-scale gene expression analysis in a limited number of patient samples, we here aimed to perform a candidate gene-based screening approach. Our basic hypothesis was that WG patients exhibit an a priori defect in epithelial barrier function of the nasal mucosa affecting innate defense mechanisms against bacterial pathogens. In consequence, in this study, we focused on genes either involved in barrier function or innate immunity or on genes with proven or potential contribution to WG pathology. We chose 49 target genes, which belong to one of the following functional groups: innate immune receptors (TLRs, NLRs, peptidoglycan-recognition receptor, and scavenger receptor (CD36)), adaptors of TLR or NLR signaling cascades, AMPs, cytokines involved in epithelial immune responses, and extracellular matrix proteins. In addition, PR3 and PAR-2 were chosen based on their well-established involvement in WG pathogenesis. ${ }^{2}$ For a complete list of target genes, see Supplementary Information online.

Gene expression profiling using real-time PCR. The mRNA expression levels of the 49 selected target transcripts and 3 housekeeping genes (ACTB, GAPDH, and 18S) were analyzed in duplicate and quantified by TaqMan Low-Density Array Real-Time PCR (Applied Biosystems). In brief, $35 \mu \mathrm{l}$ of single-stranded cDNA (equivalent to $35 \mathrm{ng}$ of total cellular RNA) was mixed with $15 \mu$ l nuclease-free water and $50 \mu \mathrm{l}$ TaqMan Universal PCR Master Mix. The total volume of $100 \mu \mathrm{l}$ was loaded into each sample port of the Micro Fluidic Cards that were subsequently sealed and centrifuged at $256 \mathrm{~g}$ for $10 \mathrm{~min}$. Each card was analyzed on the ABI $7900 \mathrm{HT}$ platform (Applied Biosystems). The thermal cycling conditions were $2 \mathrm{~min}$ at $50^{\circ} \mathrm{C}$ and $10 \mathrm{~min}$ at $95^{\circ} \mathrm{C}$, followed by 40 cycles of $30 \mathrm{~s}$ at $97^{\circ} \mathrm{C}$ and $1 \mathrm{~min}$ at $60^{\circ} \mathrm{C}$. A complete list of the genes analyzed and respective real-time PCR assays used in this study is provided in Supplementary Table S2 online.

Real-time PCR data analysis. Acquired real-time PCR data were analyzed using the $2^{-\Delta \Delta \mathrm{Ct}}$ method. ${ }^{52}$ Fold changes were calculated based on the ratio between WG samples and NC individuals, and to determine significant expression differences between the two groups, the non-parametric Mann-Whitney $U$-test was used. The obtained $P$-value was adjusted for multiple testing using the Benjamini-Hochberg correction based on all detected transcripts $(n=43) .{ }^{53}$ Corrected $P$-values $P \leqslant 0.05$ were considered significant. To determine the FDR of fold changes, a Westfall and Young ${ }^{54}$ permutation ( $k=10,000$ permutations) was performed for each gene individually. Fold changes with a FDR $\leqslant 5 \%$ were considered significant. Only transcripts meeting both cutoff criteria $(P \leqslant 0.05$ and FDR $\leq 5 \%)$ were considered significant and potentially disease relevant. Cutoff boundaries for fold changes were determined for each gene individually by assessing at which fold change the FDR would be $>5 \%$ (based on the Westfall and Young permutation).

Addressing potential confounding factors. To ensure that large-scale infiltration of immune cells in the biopsies does not increase sample heterogeneity, all target areas were macroscopically inspected for the lack of signs of inflammation before taking the biopsy. In addition, different cell compositions of biopsies and cell heterogeneity in each biopsy were addressed by applying non-parametric methods for the statistics and by using larger sample numbers. Individual patient traits which may potentially influence mRNA expression levels were evaluated for their confounding impact by a substratification analysis.

Cluster analysis. To generate a functional profile of differences between WG and NCs, a cluster of transcripts meeting the cutoff criteria for significance was created and displayed as a heatmap (clustering method: UPGMA (unweighted pair grouped method with arithmetic mean), distance measure: correlation, software: Spotfire DSMA 9.1 (TIBCO, Palo Alto, CA)).

Principal component analysis. Principal component analysis was performed after $z$-score normalization using Spotfire DSMA 9.1.2.

Correlation analysis. To identify co-regulated genes, a similarity analysis was performed using Spearman's-rho correlation coefficient, comparing each transcript with all other transcripts. The obtained correlation coefficients were clustered as described above and displayed as a heatmap.

SDS-PAGE and immunoblotting. Snap-frozen biopsies were lysed in $1 \times$ lysis buffer $\left(1 \%\right.$ SDS, $10 \mathrm{~mm}$ Tris $\left.\mathrm{pH} 7.6,1 \mathrm{~mm} \mathrm{Na}_{3} \mathrm{VO}_{4}\right)$ containing phosphatase and protease inhibitor cocktails (Sigma-Aldrich, St Louis, $\mathrm{MO})$, followed by boiling ( $5 \mathrm{~min}$ ) and sonification. Lysates were cleared from debris by centrifugation. Overall, $10 \mu \mathrm{g}$ of cleared protein extracts 
was separated by denaturing SDS-PAGE and transferred onto polyvinylidene fluoride membranes (Millipore, Billerica, MA). After blocking, membranes were probed with the following specific primary antibodies: rabbit anti-NLRC3 (1:250, Abcam, Cambridge, MA), rabbit anti-TLR4 (1:200, Santa Cruz Biotechnology, Santa Cruz, CA), rabbit anti-CD36 (1:200, Cayman Chemical Company, Ann Arbor, MI). After extensive washing, membranes were incubated with the corresponding horseradish peroxidase-conjugated secondary antibody (Amersham Biosciences, Piscataway, NJ). Proteins were visualized by enhanced chemiluminescence (Amersham Biosciences). To determine even transfer and equal loading, membranes were stripped and reprobed with mouse anti- $\beta$-actin antibody (1:1,000, Sigma-Aldrich).

Immunohistochemistry. For antigen retrieval, 4- $\mu$ m-thick formalinfixed, paraffin-embedded biopsies were deparaffinized, rehydrated, and microwave treated in $0.01 \mathrm{M}$ citrate buffer $(\mathrm{pH}$ 6.0). Endogenous peroxidase was blocked by $0.3 \% \mathrm{H}_{2} \mathrm{O}_{2}$ for $10 \mathrm{~min}$ and the slides were incubated with 3\% normal rabbit serum (for subsequent detection of S100A7) and normal swine serum (for TLR4) (Vector, Burlingame, CA) to avoid nonspecific binding. After blocking, slides were incubated with polyclonal rabbit anti-human TLR4 antibody ( $3 \mu \mathrm{g} \mathrm{ml}$, Santa Cruz Biotechnology, Heidelberg, Germany) or with murine monoclonal anti-human-S100A7 antibody (HL15-4, $1.25 \mathrm{\mu g} \mathrm{ml}^{-1}$, kindly provided by $\mathrm{R}$ Gläser and $\mathrm{H}$ Lange, Kiel, Germany) for $1 \mathrm{~h}$ at room temperature or overnight at $4{ }^{\circ} \mathrm{C}$. Slides were rinsed with Tris-buffered saline and incubated for $30 \mathrm{~min}$ with the appropriate biotinylated secondary antibodies at room temperature. For S100A7, polyclonal rabbit anti-mouse immunoglobulins $\left(6.5 \mu \mathrm{g} \mathrm{ml}^{-1}\right.$, Dako, Glostrup, Denmark) and for TLR4, polyclonal swine anti-rabbit immunoglobulins $\left(4.4 \mu \mathrm{g} \mathrm{ml}^{-1}\right.$, Dako) were applied. Slides were incubated using streptavidin-peroxidase reagent "Vector ABC kit" (Vector) for $30 \mathrm{~min}$ after washing with Tris-buffered saline. The activity of peroxidase was detected using "Vector NovaRed Substrate kit" (Vector) and biopsies were counterstained with hematoxylin. Tonsil biopsies served as positive controls..$^{55,56}$ Negative controls were processed identically omitting primary antibodies, and hematoxylin staining was performed in parallel to visualize tissue architecture and cell distribution.

SUPPLEMENTARY MATERIAL is linked to the online version of the paper at http://www.nature.com/mi

\section{ACKNOWLEDGMENTS}

We thank $R$ Gläser and $H$ Lange for valuable reagents. A Hölzgen, $U$ Kreutz, D Oelsner, T Kaacksteen, M Schlapkohl, A Graeff, T Görögh, and A-M Röen are gratefully acknowledged for their expert technical assistance. We deeply acknowledge the participation of all patients and healthy volunteers who took part in this study. This work was supported by grants from the German Research Foundation (DFG) funded Clinical Research Unit/KFO 170, the Excellence Cluster "Inflammation at Interfaces," and the National Genome Research Network (NGFN plus).

\section{DISCLOSURE}

The authors declared no conflict of interest.

C) 2011 Society for Mucosal Immunology

\section{REFERENCES}

1. Reinhold-Keller, E., Herlyn, K., Wagner-Bastmeyer, R. \& Gross, W.L. Stable incidence of primary systemic vasculitides over five years: results from the German Vasculitis Register. Arthritis Rheum. 53, 93-99 (2005).

2. Lamprecht, P. \& Gross, W.L. Current knowledge on cellular interactions in the WG-granuloma. Clin. Exp. Rheumatol. 25, S49-S51 (2007).

3. Laudien, M., Ambrosch, P., Till, A., Podschun, R. \& Lamprecht, P. [Diagnosis, therapy and current research aspects of selected chronic inflammatory diseases with head and neck involvement]. Z. Rheumatol. 67, 397-406 (2008).

4. Wegener, F. Über eine eigenartige rhinogene Granulomatose mit besonderer Beteiligung des Arteriensystems und der Nieren. Beitr. Pathol. Anatom. 102, 36-68 (1939).
5. Laudien, M. et al. Nasal carriage of Staphylococcus aureus and endonasal activity in Wegener $\mathrm{s}$ granulomatosis as compared to rheumatoid arthritis and chronic rhinosinusitis with nasal polyps. Clin. Exp. Rheumatol. 28, 51-55 (2010).

6. Popa, E.R. et al. Staphylococcal toxic-shock-syndrome-toxin-1 as a risk factor for disease relapse in Wegener's granulomatosis. Rheumatology (Oxford) 46, 1029-1033 (2007).

7. Stegeman, C.A. et al. Association of chronic nasal carriage of Staphylococcus aureus and higher relapse rates in Wegener granulomatosis. Ann. Intern. Med. 120, 12-17 (1994).

8. van Putten, J.W., van Haren, E.H. \& Lammers, J.W. Association between Wegener's granulomatosis and Staphylococcus aureus infection? Eur. Respir. J. 9, 1955-1957 (1996).

9. Popa, E.R., Stegeman, C.A., Kallenberg, C.G. \& Tervaert, J.W. Staphylococcus aureus and Wegener's granulomatosis. Arthritis Res. 4, 77-79 (2002).

10. Jagiello, P., Gross, W.L. \& Epplen, J.T. Complex genetics of Wegener granulomatosis. Autoimmun. Rev. 4, 42-47 (2005).

11. Schreiber, S., Rosenstiel, P., Albrecht, M., Hampe, J. \& Krawczak, M. Genetics of Crohn disease, an archetypal inflammatory barrier disease. Nat. Rev. Genet. 6, 376-388 (2005).

12. Swidsinski, A. et al. Mucosal flora in inflammatory bowel disease. Gastroenterology 122, 44-54 (2002).

13. Mayet, W.J., Marker-Hermann, E., Schlaak, J. \& Meyer zum Buschenfelde, K.H. Irregular cytokine pattern of CD4+ T lymphocytes in response to Staphylococcus aureus in patients with Wegener's granulomatosis. Scand. J. Immunol. 49, 585-594 (1999).

14. Israel, H.L. Sulfamethoxazole-trimethoprim therapy for Wegener's granulomatosis. Arch. Intern. Med. 148, 2293-2295 (1988).

15. Rasmussen, N., Petersen, J., Remvig, L. \& Andersen, V. Treatment of Wegener's granulomatosis with trimethoprim-sulfamethoxazole. APMIS Suppl. 19, 61-62 (1990).

16. Rusterholz, D. \& Schlegel, C. [Infectious aspects of Wegener's granulomatosis]. Schweiz. Med. Wochenschr. (Suppl 125), 41S-43S (2000).

17. Stegeman, C.A., Tervaert, J.W., De Jong, P.E., Kallenberg, C.G., \& Dutch Co-Trimoxazole Wegener Study Group Trimethoprim-sulfamethoxazole (co-trimoxazole) for the prevention of relapses of Wegener's granulomatosis.. N. Engl. J. Med. 335, 16-20 (1996).

18. De Groot, K. et al. Therapy for the maintenance of remission in sixty-five patients with generalized Wegener's granulomatosis. Methotrexate vs. trimethoprim/sulfamethoxazole. Arthritis Rheum. 39, 2052-2061 (1996).

19. Reinhold-Keller, E. et al. Response to trimethoprim/sulfamethoxazole in Wegener's granulomatosis depends on the phase of disease. QJM 89, 15-23 (1996).

20. Vaudaux, P., Kelley, W.L. \& Lew, D.P. Staphylococcus aureus small colony variants: difficult to diagnose and difficult to treat. Clin. Infect. Dis. 43, 968-970 (2006).

21. DeRemee, R.A. The treatment of Wegener's granulomatosis with trimethoprim/sulfamethoxazole: illusion or vision? Arthritis Rheum. 31, 1068-1074 (1988).

22. Bernstein, J.M. Mucosal immunology of the upper respiratory tract. Respiration 59 (Suppl 3), 3-13 (1992).

23. Ullrich, S. et al. Severe impaired respiratory ciliary function in Wegener granulomatosis. Ann. Rheum. Dis. 68, 1067-1071 (2009).

24. Peyrin-Biroulet, L. et al. Peroxisome proliferator-activated receptor gamma activation is required for maintenance of innate antimicrobial immunity in the colon. Proc. Natl Acad. Sci. USA 107, 8772-8777 (2010).

25. Costello, C.M. et al. Dissection of the inflammatory bowel disease transcriptome using genome-wide cDNA microarrays. PLoS Med. 2, e199 (2005).

26. Abdulahad, W.H., Stegeman, C.A., Limburg, P.C. \& Kallenberg, C.G. Skewed distribution of Th17 lymphocytes in patients with Wegener's granulomatosis in remission. Arthritis Rheum. 58, 2196-2205 (2008).

27. Csernok, E. et al. Transforming growth factor-beta (TGF-beta) expression and interaction with proteinase 3 (PR3) in anti-neutrophil cytoplasmic antibody (ANCA)-associated vasculitis. Clin. Exp. Immunol. 105, 104-111 (1996).

28. Muller, A. \& Lamprecht, P. [Interleukin-17 in chronic inflammatory and autoimmune diseases: rheumatoid arthritis, Crohn's disease and Wegener's granulomatosis]. Z. Rheumatol. 67, 72-74 (2008).

29. Kozak, M. Some thoughts about translational regulation: forward and backward glances. J. Cell Biochem. 102, 280-290 (2007). 
30. Holmes, M.V. et al. Fulfilling the promise of personalized medicine? Systematic review and field synopsis of pharmacogenetic studies. PLoS One 4, e7960 (2009).

31. Verweij, C.L. Transcript profiling towards personalised medicine in rheumatoid arthritis. Neth. J. Med. 67, 364-371 (2009).

32. Hasler, R. et al. Genetic control of global gene expression levels in the intestinal mucosa: a human twin study. Physiol. Genomics 38, 73-79 (2009).

33. Chen, X. et al. Synergistic effect of antibacterial agents human betadefensins, cathelicidin LL-37 and lysozyme against Staphylococcus aureus and Escherichia coli. J. Dermatol. Sci. 40, 123-132 (2005).

34. Morrison, G., Kilanowski, F., Davidson, D. \& Dorin, J. Characterization of the mouse beta defensin 1, Defb1, mutant mouse model. Infect. Immun. 70, 3053-3060 (2002).

35. Frank, D.N. et al. Molecular-phylogenetic characterization of microbial community imbalances in human inflammatory bowel diseases. Proc. Natl Acad. Sci. USA 104, 13780-13785 (2007).

36. Manichanh, C. et al. Reduced diversity of faecal microbiota in Crohn's disease revealed by a metagenomic approach. Gut 55, 205-211 (2006).

37. Ott, S.J. et al. Reduction in diversity of the colonic mucosa associated bacterial microflora in patients with active inflammatory bowel disease. Gut 53, 685-693 (2004).

38. Wieczorek, S., Holle, J.U. \& Epplen, J.T. Recent progress in the genetics of Wegener's granulomatosis and Churg-Strauss syndrome. Curr. Opin. Rheumatol. 22, 8-14 (2010).

39. Wieczorek, S. et al. A functionally relevant IRF5 haplotype is associated with reduced risk to Wegener's granulomatosis. J. Mol. Med. 88, 413-421 (2010).

40. Takaoka, A. et al. Integral role of IRF-5 in the gene induction programme activated by Toll-like receptors. Nature 434, 243-249 (2005).

41. Wilson, D. et al. Identifying infection-associated genes of Candida albicans in the postgenomic era. FEMS Yeast Res. 9, 688-700 (2009).

42. Stefaniuk, P., Cianciara, J. \& Wiercinska-Drapalo, A. Present and future possibilities for early diagnosis of hepatocellular carcinoma. World $\mathrm{J}$. Gastroenterol. 16, 418-424 (2010).
43. Machugh, D.E. et al. Gene expression profiling of the host response to Mycobacterium bovis infection in cattle. Transbound. Emerg. Dis. 56, 204-214 (2009).

44. Handkiewicz-Junak, D., Czarniecka, A. \& Jarzab, B. Molecular prognostic markers in papillary and follicular thyroid cancer: current status and future directions. Mol. Cell Endocrinol. 322, 8-28 (2010).

45. Eddy, J.A., Sung, J., Geman, D. \& Price, N.D. Relative expression analysis for molecular cancer diagnosis and prognosis. Technol. Cancer Res. Treat. 9, 149-159 (2010).

46. Hellmich, B. et al. EULAR recommendations for conducting clinical studies and/or clinical trials in systemic vasculitis: focus on anti-neutrophil cytoplasm antibody-associated vasculitis. Ann. Rheum. Dis. 66, 605-617 (2007).

47. Luqmani, R.A. Assessing disease activity in the systemic vasculitides. Curr. Opin. Rheumatol. 14, 23-28 (2002).

48. DeRemee, R.A., McDonald, T.J., Harrison, E.G. Jr. \& Coles, D.T. Wegener's granulomatosis. Anatomic correlates, a proposed classification. Mayo Clin. Proc. 51, 777-781 (1976).

49. Paulsen, J.I. \& Rudert, H. [Manifestations of primary vasculitis in the ENT region]. Z. Rheumatol. 60, 219-225 (2001).

50. Reinhold-Keller, E. et al. An interdisciplinary approach to the care of patients with Wegener's granulomatosis: long-term outcome in 155 patients. Arthritis Rheum. 43, 1021-1032 (2000).

51. Benninger, M.S. et al. Adult chronic rhinosinusitis: definitions, diagnosis, epidemiology, and pathophysiology. Otolaryngol. Head Neck Surg. 129, S1-S32 (2003).

52. Livak, K.J. \& Schmittgen, T.D. Analysis of relative gene expression data using real-time quantitative PCR and the 2(-Delta Delta C(T)) method. Methods 25, 402-408 (2001).

53. Benjamini, Y. \& Hochberg, Y. A practical and powerful approach to multiple testing. J. Roy. Statist. Soc. 57, 289-300 (1995).

54. Westfall, P.H. \& Young, S. Resampling-Based Multiple Testing (WileyInterscience, New York, 1993).

55. Bryborn, M., Mansson, A., Cardell, L.O. \& Adner, M. Differentiated S100A7 expression in infected tonsils and tonsils from allergic individuals. FEMS Immunol. Med. Microbiol. 53, 413-420 (2008).

56. Claeys, S. et al. Human beta-defensins and toll-like receptors in the upper airway. Allergy 58, 748-753 (2003). 